\title{
Effects of Vacuum Annealing on Mechanical Properties of Gallium-implanted Silicon Nanowires
}

\author{
Tatsuya Fujii, ${ }^{1 *}$ Takahiro Namazu, ${ }^{2}$ Koichi Sudoh, ${ }^{3}$ and Eri Miura-Fujiwara ${ }^{4}$ \\ ${ }^{1}$ Department of Mechanical Engineering, Akita Prefectural University, \\ 84-4 Aza Ebinokuchi, Tsuchiya, Yurihonjo, Akita 015-0055, Japan \\ ${ }^{2}$ Department of Mechanical Engineering, Aichi Institute of Technology, \\ 1247 Yachigusa, Yakusa, Toyota, Aichi 470-0392, Japan \\ ${ }^{3}$ The Institute of Scientific and Industrial Research, Osaka University, \\ 8-1 Mihogaoka, Ibaraki, Osaka 567-0047, Japan \\ ${ }^{4}$ Department of Mechanical Engineering and Metallurgy, University of Hyogo, \\ 2167 Shosha, Himeji, Hyogo 671-2201, Japan
}

(Received November 9, 2018; accepted January 11, 2019)

Keywords: Si nanowires, focused ion beam, vacuum annealing, tensile test, in situ SEM, mechanical property, surface damage, crystal recovery

In this paper, we describe the effects of vacuum annealing on the mechanical properties of gallium (Ga)-implanted silicon ( $\mathrm{Si}$ ) nanowires (NWs) obtained by focused ion beam (FIB) fabrication. We have particularly developed a micro-electromechanical system (MEMS)-based tensile test device to enable us to directly apply tension to nanoscale Si NWs. The nonannealed and annealed Si NWs made from a silicon-on-nothing ( $\mathrm{SON}$ ) membrane were prepared. The Ga-implanted Si NWs, which were sampled on the MEMS device without FIB irradiation, were annealed at $700{ }^{\circ} \mathrm{C}$ for $10 \mathrm{~s}$ to $60 \mathrm{~min}$ in high vacuum. The quasi-static uniaxial tensile tests of all the NWs were conducted by in situ scanning electron microscopy (SEM). The dependences of annealing time on Young's modulus and fracture strength were observed. Young's modulus gradually recovered with increasing annealing time, whereas the strength dropped first at $10 \mathrm{~s}$ of annealing and then slightly increased with annealing time. The difference in the recovery process between these characteristics was discussed from the viewpoint of crystallinity-damage recovery.

\section{Introduction}

In recent years, with the benefit of rapidly developing semiconductor processing technology, micro/nano-electromechanical systems (MEMS/NEMS) with nanoscale mechanical structures made of silicon ( $\mathrm{Si}$ ) and its related materials have been actively researched and developed toward various industrial applications. ${ }^{(1-3)}$ Si-based nanostructures such as nanowires (NWs) and nanotubes (NTs) play important roles as mechanical and electrical components in these devices. ${ }^{(2-5)}$ The methods of manufacturing nanostructures are classified as top-down methods, as typified by micromachining techniques including the deposition and etching of thin films, and bottom*Corresponding author: e-mail: t-fujii@akita-pu.ac.jp https://doi.org/10.18494/SAM.2019.2193 
up methods, as typified by self-organization techniques including vapor-liquid-solid (VLS) synthesis. $^{(1-6)}$ Among these techniques, the focused ion beam (FIB) processing technique is one of the powerful tools for nanofabrication, and three-dimensional mechanical parts with nanosized dimensions can be manufactured directly by a combinatorial technique of FIB milling, FIB-assisted chemical vapor deposition (CVD), and microprobe sampling. ${ }^{(7-9)}$ In FIB fabrication, surface damage is definitely induced upon gallium $\left(\mathrm{Ga}^{+}\right)$ion irradiation ${ }^{(10)}$ and is expected to reduce the mechanical reliability of Si nanostructures. In our previous research, ${ }^{(11)}$ we conducted the tensile test of FIB-fabricated Si NWs and evaluated the effects of FIB-induced damage on the mechanical properties; Young's moduli of FIB-fabricated Si NWs ranged from 107.4 to $144.2 \mathrm{GPa}$, which was an approximately $14.6-36.4 \%$ reduction from the ideal value for Si (001)[110]. Therefore, to improve the reliability of FIB-fabricated Si nanostructures, surface damage should be completely removed. For removing the damaged portion, there are methods involving surface corrosion with a chemical solution, dry etching, and ion milling with low-acceleration $\mathrm{Ga}^{+}$or argon $\left(\mathrm{Ar}^{+}\right)$ion beams, but it is difficult to remove the damage completely by these processes. ${ }^{(12-14)}$ In recent research studies, a novel technique for repairing the FIB-induced damage by vacuum annealing has been proposed and has attracted attention. ${ }^{(15-18)}$ This technique may enable the recovery of the crystallinity of the damaged portion by evaporating the $\mathrm{Ga}^{+}$ions implanted into the crystalline material; this heat treatment may improve the mechanical properties of FIB-fabricated Si nanostructures. Schilling et al. investigated the vacuum annealing effect of FIB-fabricated single-crystal barium titanate $\left(\mathrm{BaTiO}_{3}\right)$ nanostructures and showed that the implanted Ga can be removed while forming island-shaped Ga-rich platelets on a $\mathrm{BaTiO}_{3}$ thin-film surface. ${ }^{(17)}$ Ishida et al. also showed that Young's moduli of tungsten nanostructures grown by FIB-CVD using tungsten hexacarbonyl [W(CO) 6 ] are recovered by vacuum annealing. ${ }^{(18)}$ Furthermore, we demonstrated that the FIB damage can be removed by $700{ }^{\circ} \mathrm{C}$ annealing in high vacuum. ${ }^{(19)}$ However, during annealing, since Si atoms freely move and diffuse on the surface, the best conditions for completely suppressing the crystallinity damage and annihilation of the implanted Ga must be specified to achieve the mechanical/structural uniformity and reliability of annealed Si nanostructures.

In this research, we focus on the effects of vacuum annealing on the mechanical properties of Ga-implanted Si NWs. The quasi-static tensile tests for two types of Si NWs, FIB-fabricated and $700-^{\circ} \mathrm{C}$-annealed Si NWs, were carried out to examine the mechanical properties. Then, the relationship between FIB-induced damage and vacuum annealing was investigated through scanning electron microscopy (SEM) observation, energy-dispersive X-ray (EDX) analysis, cross-sectional transmission electron microscopy (TEM) observation, and structural analysis by electron diffraction.

\section{Experimental Procedure}

A MEMS-based tensile test device was specially developed for the direct mechanical evaluation of Si NWs. ${ }^{(20)}$ In this research, we decided to prepare Si NW samples separately from the test device, so that we can conduct the tensile tests of a large number of samples using only one device. This device was fabricated using conventional micromachining technologies. 
Figures 1(a)-1(f) show the process of fabricating the MEMS-based tensile test device. The starting substrate was a silicon-on-insulator (SOI) wafer with a $35-\mu \mathrm{m}$-thick single-crystal $\mathrm{Si}$ (SCS) device layer and a 2- $\mu \mathrm{m}$-thick buried oxide (BOX) layer on a $200-\mu \mathrm{m}$-thick SCS handle layer [see Fig. 1(a)]. First, 30-nm-thick chromium (Cr) and 300-nm-thick gold (Au) thin films were deposited on the top surface by sputtering, and the electrode for wire bonding was patterned [see Fig. 1(b)]. Then, a 100-nm-thick aluminum (Al) thin film was coated on the top surface by sputtering, and the electrostatic actuator and capacitive sensor patterns were formed as a metal mask for deep reactive ion etching (DRIE). The back surface was then patterned with a 200-nm-thick Al thin film in a similar manner [see Fig. 1(c)]. Subsequently, both wafer surfaces were etched by DRIE with a BOSCH process recipe [see Fig. 1(d)]. The remaining Al layers were removed [see Fig. 1(e)]. The exposed BOX layer was finally removed using a vaporized hydrofluoric (HF) solution to release the movable parts of the device [see Fig. 1(f)].

Figure 2 shows the SEM images of the produced MEMS tensile test device. This device consists of many comblike structures used as a comb-drive electrostatic actuator for applying uniaxial tensile force, a capacitive active sensor for measuring displacement, and a capacitive driven sensor for measuring displacement and force. All the comb structures are supported by suspension beams, which can freely move toward the in-plane tensile direction only. The sample stage is located between the two capacitive sensors. Each functional element for the nanoscale tensile test was fabricated well, and the sidewalls with a 1- $\mu$ m-pitch scallop pattern were finely etched by DRIE. A Si NW sample was attached to the two stages similarly to a nanobridge. ${ }^{(11,19)}$ The elongation of the NW specimen was obtained from the difference between the two displacement sensor signals derived from capacitive changes. The capacitance of comb structures was measured using two LCR meters (Keysight Technologies Inc., E4980A) with the precision of the aF order. The tensile force was calculated by multiplying the

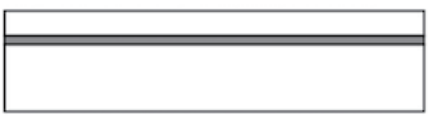

(a)

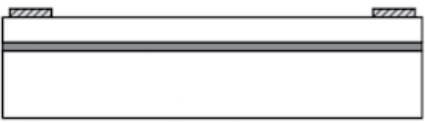

(b)

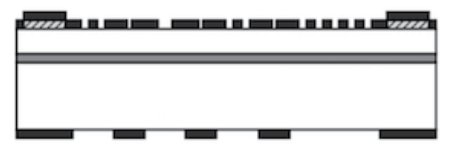

(c)

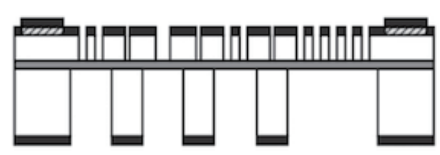

(d)

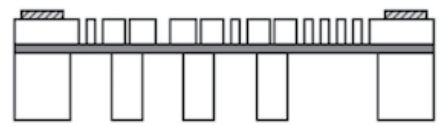

(e)

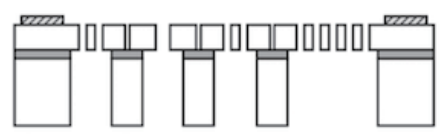

(f)

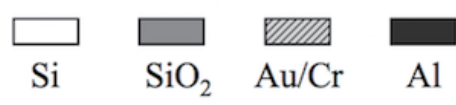

Fig. 1. Process of fabricating the MEMS-based tensile test device: (a) SOI wafer with a $35-\mu \mathrm{m}$-thick device layer, (b) fabrication of the $\mathrm{Au} / \mathrm{Cr}$ electrode, (c) patterning of Al layers on both surfaces, (d) DRIE process, (e) removal of Al layers, and (f) HF vapor etching of the sacrificed layer. 


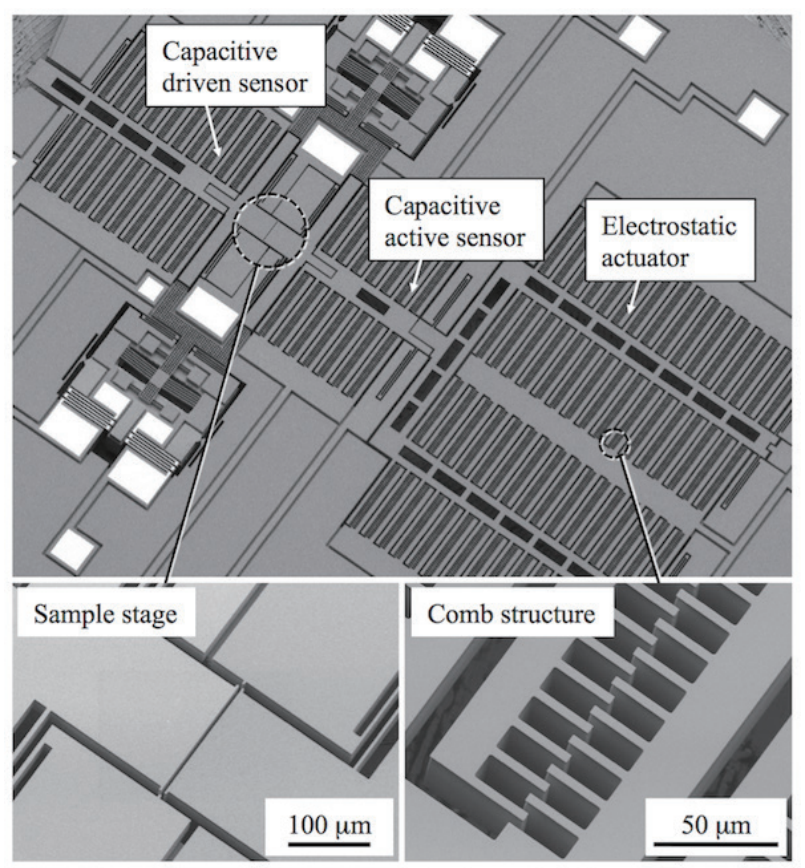

Fig. 2. SEM images of the MEMS device fabricated for nanoscale tensile tests. This device consists of a combdrive electrostatic actuator, a capacitive displacement sensor, a capacitive force sensor, and a sample stage.

displacement by the spring constant of the suspension beams of a driven sensor. The spring constant was directly calibrated using a commercial microforce-sensing probe (FemtoTools AG, FT-S1000-LAT, resolution $=0.05 \mu \mathrm{N}$ ).

We prepared two types of Si NWs, namely, nonannealed and annealed Si NWs, to examine the effects of vacuum annealing on the mechanical properties. Figure 3(a) shows the process of fabricating the nonannealed Si NWs. The starting material for preparing the NW samples was a silicon-on-nothing (SON) membrane with a self-standing SCS nanomembrane on a cavity. The SON membrane had an extremely flat surface and an excellent crystallinity produced by the high-temperature annealing of the high-aspect-ratio hole array fabricated on a $\mathrm{Si}(001)$ surface. $^{(21,22)}$ SON-membrane-derived Si NWs were fabricated using FIB-related techniques. First, square cutoff lines were fabricated by FIB milling, and a membrane sheet was lifted off using a microsampling probe. A tungsten (W) film prepared by FIB-assisted CVD was used as an adhesive material to bond the probe to the sheet. Subsequently, the lifted sheet was moved immediately above the center of the two sample stages in the device, and $\mathrm{W}$ films were deposited to attach the sheet to these stages. Finally, the sheet was FIB-fabricated at $40 \mathrm{kV}$ to form Si NWs. The top surface and sidewalls of the Si NWs suffered from FIB-induced damage because they were attacked by $\mathrm{Ga}^{+}$ions during fabrication. ${ }^{(11)}$ To prevent such damage as much as possible, we conducted high-vacuum annealing at $700{ }^{\circ} \mathrm{C}$, which led to the preparation of annealed Si NWs, as shown in Fig. 3(b). First, a SON membrane was fabricated by FIB milling to form Si NWs and lifted using a microprobe. Then, the cassette-shaped sample holder was 


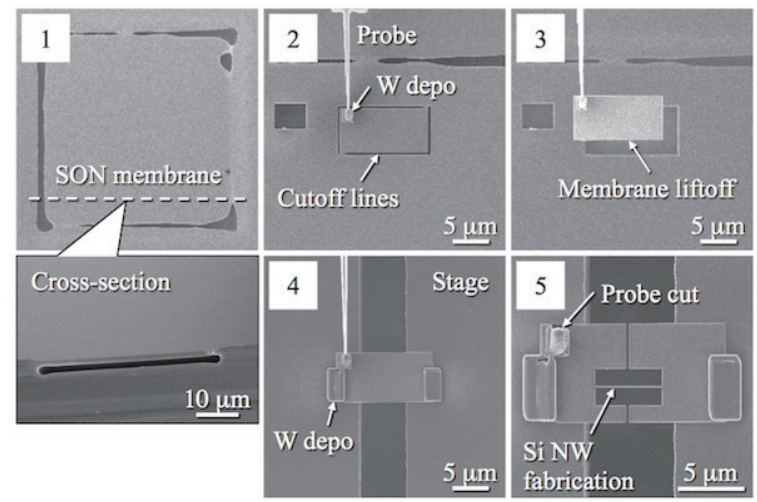

(a)
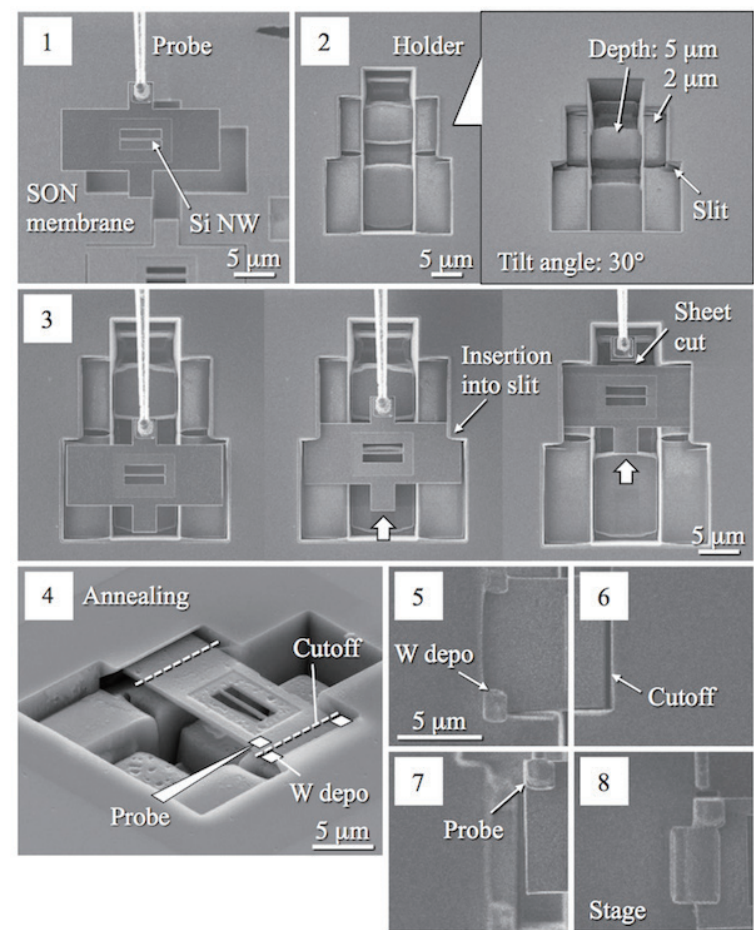

(b)

Fig. 3. Process of fabricating two types of Si NWs obtained from a SON membrane: (a) FIB-fabricated Si NW and (b) $700-{ }^{\circ} \mathrm{C}$-annealed $\mathrm{Si}$ NW. The longitude direction was set to be along the [110] direction.

FIB-fabricated on a Si substrate to fix and anneal the membrane sheet with a Si NW. This holder had two slit structures fabricated at an angle of $45^{\circ}$ and the NW sample was fixed by inserting it from the side of this holder along the slit. Finally, the edge of the sheet bonded to the probe was FIB-cut. By these processes, we were able to fix the sample without using W film, which may chemically react with Si during annealing. The NWs fixed to the holder were annealed at $700{ }^{\circ} \mathrm{C}$ in high vacuum for $10 \mathrm{~s}, 1 \mathrm{~min}, 5 \mathrm{~min}, 10 \mathrm{~min}$, and $60 \mathrm{~min}$. After annealing, the annealed Si NWs were removed from the holder and attached to the device by FIB-CVD. During this process, the NWs were sampled without FIB observation, so it is considered that the NWs do not induce further damage.

In this paper, the width and thickness of the nonannealed Si NWs were varied within the ranges from 57 and $121 \mathrm{~nm}$ to 221 and $444 \mathrm{~nm}$, respectively. These annealed Si NWs ranged from 137 and $187 \mathrm{~nm}$ to 235 and $447 \mathrm{~nm}$, respectively. The length almost corresponding to the gap between two sample stages was maintained at approximately $5.0 \mu \mathrm{m}$ in all the NWs. The longitudinal direction corresponded to the [110] direction in the (001) plane. All the nanoscale tensile tests were performed at ambient temperature in the in situ SEM system. By applying a ramping voltage up to $40 \mathrm{~V}$ to a comb-drive electrostatic actuator, a tensile force was applied to a sample until failure. To carefully measure the elongation during the tests, we simultaneously used a digital image analysis technique and a capacitance sensing technique. 


\section{Experimental Results and Discussion}

To completely repair the FIB-induced damage by high-vacuum annealing, we attempted to optimize the annealing conditions for FIB-fabricated Si NWs. In this research, we carried out annealing while changing only the holding time that we defined as the annealing time, at a constant chamber pressure of $1 \times 10^{-4} \mathrm{~Pa}$ and an annealing temperature of $700{ }^{\circ} \mathrm{C}$. Here, the selected annealing temperature was slightly higher than the recrystallization temperature of amorphous $\mathrm{Si}$, because the NWs are broken by the surface atomic diffusion of $\mathrm{Si}$, which rapidly accelerates at high temperatures. Also, the heating and cooling times were 5 and 60 min, respectively. Figure 4 shows the change in Ga percentage in NW samples with the annealing time, as measured by SEM-EDX. The Ga percentage before annealing was 27.4 at.\%, which indicates that the implanted $\mathrm{Ga}$ atoms remain in the damaged portion. With increasing annealing time, the Ga percentage rapidly decreases. This indicates that the number of implanted $\mathrm{Ga}$ atoms was definitely reduced by high-vacuum annealing. After annealing for 60 min, no Ga was detected, suggesting that $\mathrm{Ga}$ atoms were completely vaporized by annealing.

Figure 5 shows the representative tensile stress-strain relationships for Si NWs before and after high-vacuum annealing at $700{ }^{\circ} \mathrm{C}$ for $60 \mathrm{~min}$. The gray and black plots are indicative of the experimentally obtained data for the nonannealed and annealed Si NWs, respectively. The solid lines indicate the approximate lines obtained by first-order least-squares estimation in the respective stress-strain relationships. Both the Si NWs showed an almost linear stress-strain relationship until fracture, indicating that the NWs fractured in a brittle manner during elastic deformation. Young's modulus for the nonannealed Si NWs calculated from the slope of the approximate line was $139.2 \mathrm{GPa}$, which is $17.6 \%$ lower than the ideal value of $168.9 \mathrm{GPa}$, for SCS (001)[110]. ${ }^{(23)}$ In our previous study, we found that a reduction in Young's modulus is due to the effect of the FIB-induced damage layer, which is composed of amorphous $\mathrm{Si}^{(11)}$ The fracture strength was $6.8 \mathrm{GPa}$, which is 12.8 times higher than that of millimeter-sized SCS

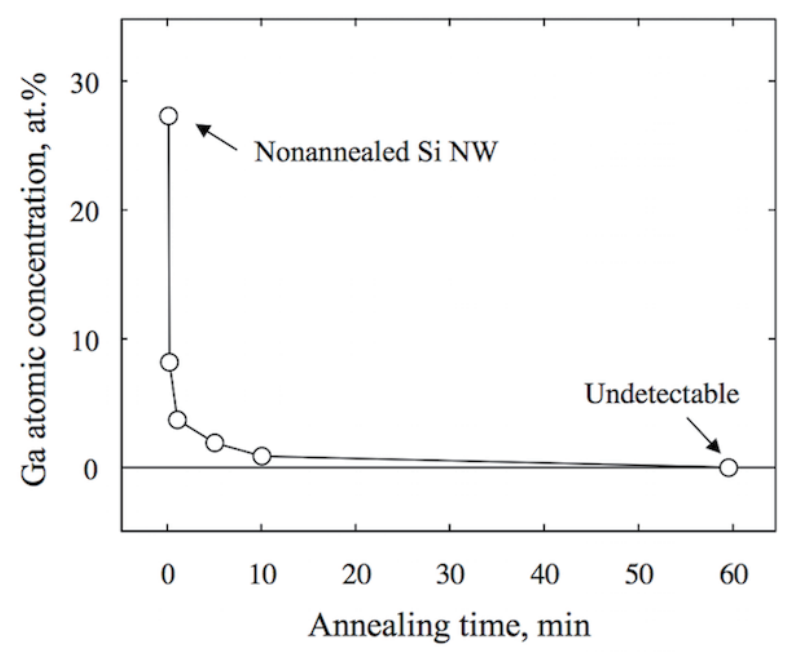

Fig. 4. Change in Ga percentage measured by EDX in NW samples with annealing time at $700{ }^{\circ} \mathrm{C}$ in high vacuum. 


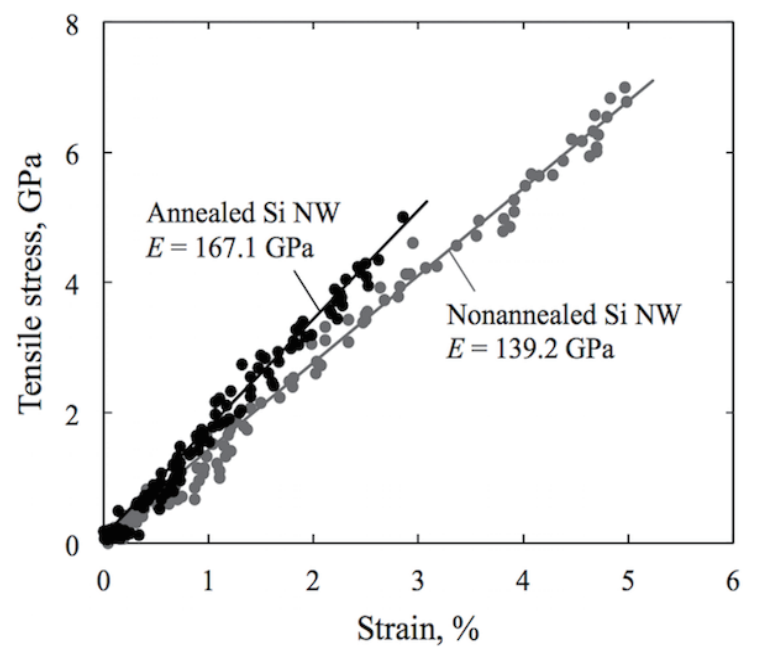

Fig. 5. Representative tensile stress-strain relationships for Si NWs before and after annealing, obtained by in situ tensile tests in SEM.

structures. $^{(24,25)}$ Therefore, it can be considered that the fracture strength of SCS has a large specimen size dependence. In the annealed Si NWs, Young's modulus increased to $167.1 \mathrm{GPa}$, which is close to the ideal value. The fracture strength was $5.0 \mathrm{GPa}$, which is $26.5 \%$ lower than that obtained before annealing. These results suggest that some structural change was induced in the FIB-damaged layer by annealing because the Young's modulus is a mechanical property based on the atomic force and distance for materials.

We conducted the tensile tests while changing the annealing time to estimate the effects of annealing on the mechanical properties of the FIB-fabricated Si NWs. Figure 6(a) shows the relationship between the annealing time and Young's modulus of the Si NWs. The tensile tests at each annealing time were carried out for more than three NWs, and the plots and error bars indicate the mean values and the variations in measured values, respectively. The data measured at the annealing time of 0 min indicate the test results for the nonannealed Si NWs. Young's modulus of the nonannealed Si NWs was $132.9 \mathrm{GPa}$ on average, which is $21.3 \%$ lower than the ideal value for SCS. After high-vacuum annealing at $700{ }^{\circ} \mathrm{C}$ for $10 \mathrm{~s}$, the mean value increased to $138.1 \mathrm{GPa}$, which is $3.9 \%$ higher than that before annealing. Upon increasing annealing time to 1 and $5 \mathrm{~min}$, the mean value gradually increased to 149.3 and $164.3 \mathrm{GPa}$, respectively. The value of the 5-min-annealed $\mathrm{Si} \mathrm{NW}$ is almost the same as the ideal value. The data dispersion of Young's moduli of the nonannealed Si NWs was found to be the largest. Figure 6(b) shows the relationship between the annealing time and the fracture strength of the Si NWs. The trend of the fracture strength was different from that of Young's modulus. The fracture strength of the nonannealed Si NWs was $5.7 \mathrm{GPa}$ on average, which is 12.1 times higher than that of the millimeter-sized SCS structures. After annealing for only $10 \mathrm{~s}$, the mean value drastically dropped to $2.8 \mathrm{GPa}$, which is $50.9 \%$ lower than that before annealing. Upon increasing annealing time to 1 and $5 \mathrm{~min}$, the mean value gradually increased to 3.2 and 3.4 GPa, respectively. However, even after annealing for $60 \mathrm{~min}$, the mean strength did not 


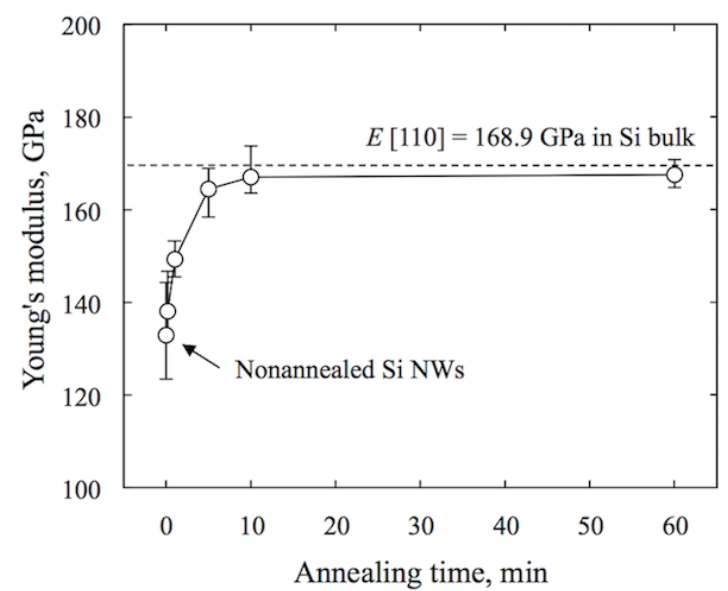

(a)

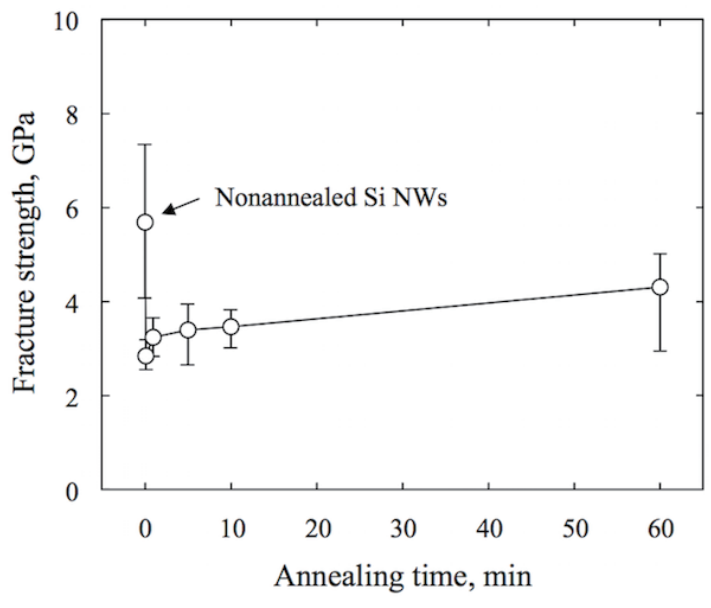

(b)

Fig. 6. Effects of annealing time on the mechanical properties of FIB-fabricated Si NWs: (a) Young's modulus and (b) fracture strength.

return to the initial value of the nonannealed Si NWs. Regarding the data dispersion of the fracture strength, the dispersion for nonannealed Si NWs was the largest, as was the dispersion of Young's modulus.

To estimate the cause of the annealing time dependence of the mechanical properties, the SEM and TEM observations of the nonannealed and annealed Si NWs were conducted. Figure 7 shows the representative top surface photographs of the Si NWs obtained by SEM. In the nonannealed Si NWs, an extremely smooth surface can be seen. By annealing at $700{ }^{\circ} \mathrm{C}$ for $10 \mathrm{~s}$, the Si NW surface became rough. This phenomenon was never seen on the annealed pure Si surface without FIB fabrication, so the rough surface was probably caused by FIB-induced $\mathrm{Ga}^{+}$ion implantation. This surface morphology gradually planarized with increasing annealing time, and it can be seen that almost nothing could be confirmed on the surface after annealing for $60 \mathrm{~min}$. These nanoscale surface asperities are considered to reduce the tensile strength of the Si NWs, because the tensile stress concentrates at these surface defects during the tensile tests.

Figures 8(a) and 8(b) show the representative cross-sectional TEM images along with electron diffraction images of the FIB-irradiated Si substrate before and after high-vacuum annealing at $700{ }^{\circ} \mathrm{C}$ for $60 \mathrm{~min}$. The TEM samples with an ultrathin section less than $100 \mathrm{~nm}$ thick were prepared by FIB-based sampling techniques. ${ }^{(10,26)}$ The starting substrate was a SCS chip. It was irradiated with $\mathrm{Ga}^{+}$ion beams at $40 \mathrm{kV}$ and annealed under predetermined conditions. First, a 150-nm-thick platinum (Pt) thin film was mild-coated on the FIB-irradiated surface; this is effective for preventing the introduction of FIB damage into the observation area during TEM sample preparation. Then, the microsample with the observation area was removed from the Si substrate and fixed on a TEM grid. Finally, the sample was FIB-thinned. Before annealing, the FIB-induced damaged portion with a brighter contrast than the undamaged $\mathrm{Si}$ substrate was observed on the top surface. The electron diffraction pattern indicates that 


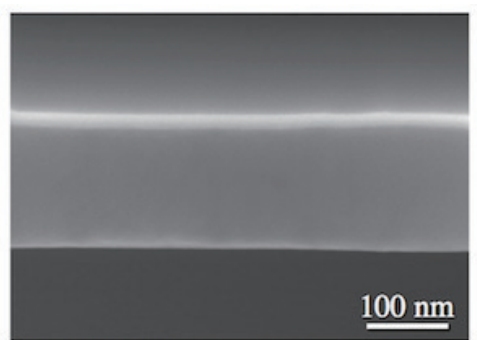

(a)

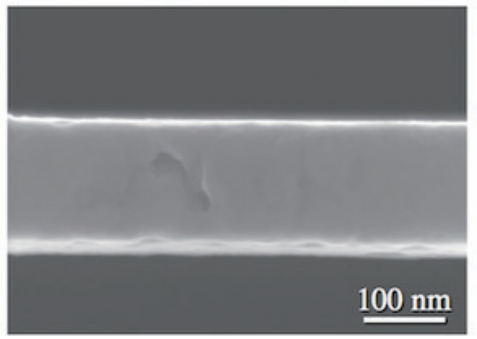

(c)

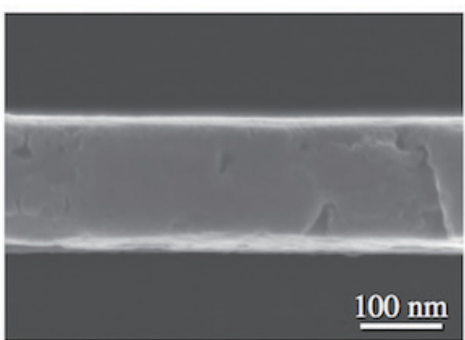

(b)

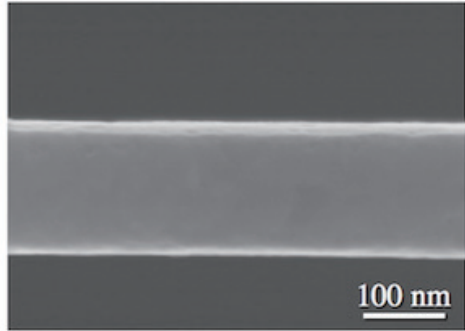

(d)

Fig. 7. SEM images of Si NW surfaces: (a) FIB-fabricated Si NW, and Si NWs annealed for (b) $10 \mathrm{~s}$, (c) 5 min, and (d) 60 min.

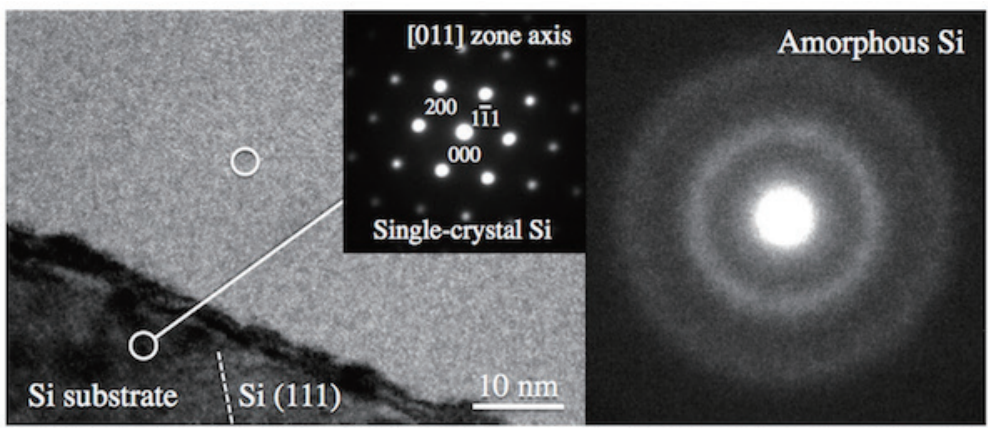

(a)

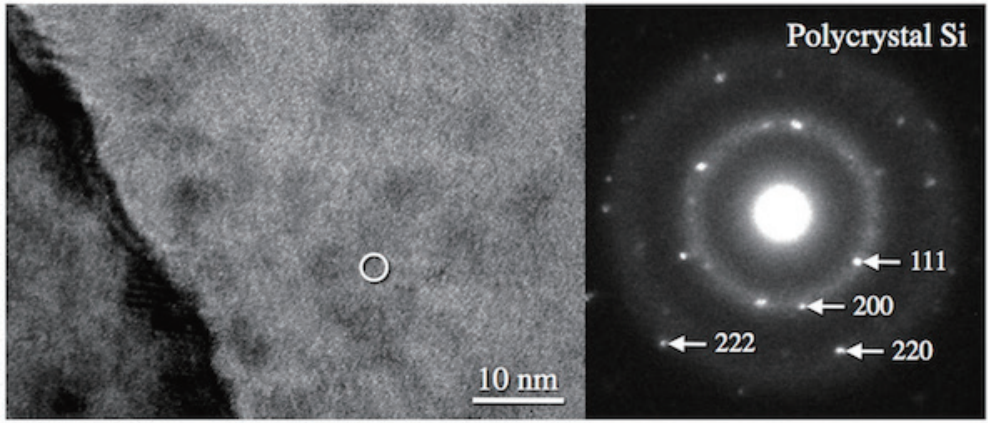

(b)

Fig. 8. TEM images (left) and diffraction spot patterns (right) of Si substrates: (a) FIB-fabricated Si substrate and (b) Si substrate annealed for $60 \mathrm{~min}$. The spot patterns of both samples correspond to the zone axis along the [011] direction. 
the crystal structures of the $\mathrm{Si}$ substrate and FIB-damaged portion are single-crystal and amorphous, respectively. We confirmed that the amorphous structure caused the small Young's moduli seen in Figs. 5 and 6. Also in the TEM image after annealing, the damaged layer was observed, but the contrast of this layer showed a patchy pattern with dark and light gray spots. The difference in contrast is due to the scattering of electrons by the diffraction phenomenon, suggesting that the damaged layer has a polycrystalline structure. From the diffraction pattern in this layer, the crystallinity of the damaged layer was recovered for polycrystal $\mathrm{Si}$. It is known that Young's modulus of poly-Si is about $160 \mathrm{GPa}$, which is higher than that of amorphous $\mathrm{Si}^{(27)}$ Thereby, the recrystallization of the FIB-induced damaged layer is thought to have increased Young's modulus.

To investigate the process of recovering the crystal structure by annealing, we made a detailed observation of the TEM sample at each annealing time. Figures 9(a)-9(d) show the TEM images near the interface between the FIB-damaged portion and the SCS substrate. As mentioned above, in the nonannealed sample, the damaged portion is amorphous, whereas the undamaged portion is single crystalline. After annealing for $10 \mathrm{~s}$, the microcrystals with a lattice fringe are partially formed in the damaged portion near the undamaged Si substrate interface. The fine crystal grains gradually spread to the entire damaged portion with increasing annealing time and, in the 5-min-annealed sample, the damaged portion was almost completely recrystallized. This suggests that the FIB-induced surface damage was gradually recrystallized from the interface near the SCS substrate upon high-vacuum annealing at $700{ }^{\circ} \mathrm{C}$. Therefore, in the case of FIB-implanted Si NWs without a pure-Si core, the crystal nucleation and growth due to the recrystallization of $\mathrm{Si}$ are considered to be restrained. Also, after annealing for $10 \mathrm{~s}$, numerous Moiré fringe patterns were formed in the damaged portion near the undamaged Si substrate interface, as indicated by white arrows in Fig. 9. This Moiré fringe gradually decreased with increasing annealing time, but a faint fringe could be confirmed on the damaged portion after annealing for $60 \mathrm{~min}$. This means that, at the damaged-undamaged boundary, large crystal misorientations definitely remained. ${ }^{(28,29)}$

From the above experimental results, we summarized the effects of annealing on the mechanical properties of Ga-implanted Si NWs shown in Fig. 10. In nonannealed Si NWs, the top surface and the sidewalls suffered from FIB-induced damage because these surfaces were attacked by $\mathrm{Ga}^{+}$ions during the fabrication. According to our previous research, ${ }^{(11,19)}$ the thicknesses of the damaged layer produced by FIB milling at $40 \mathrm{kV}$ at the top surface and sidewalls of the Si NW are around 80 and $30 \mathrm{~nm}$, respectively. During annealing, the recrystallization of $\mathrm{Si}$ in the FIB-damaged amorphous portion and the migration of $\mathrm{Ga}$ atoms to the neighboring NW surface occurred. These migrating $\mathrm{Ga}$ atoms formed $\mathrm{Ga}$ droplets and roughened the surface. Also, in the recrystallized damaged layer, the microcrystals were found to have grown from the interface near the SCS substrate. The crystal growth area steadily increased with increasing annealing time. However, even though successive annealing was performed, the damaged layer recrystallized to not SCS but polycrystalline Si. This means that, at the damaged-undamaged boundary, large crystal misorientations definitely remained. The misorientations definitely generated a large strain, thereby inducing crack initiation at the boundary. The recrystallization to poly-Si is the reason why Young's modulus increased to 


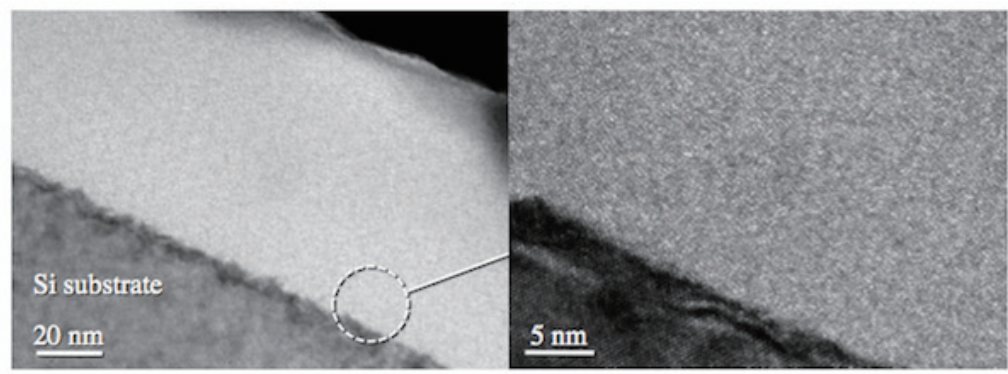

(a)

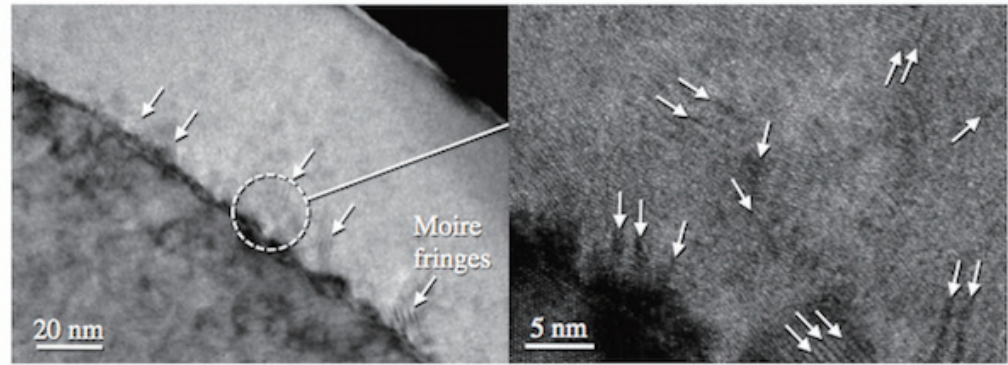

(b)

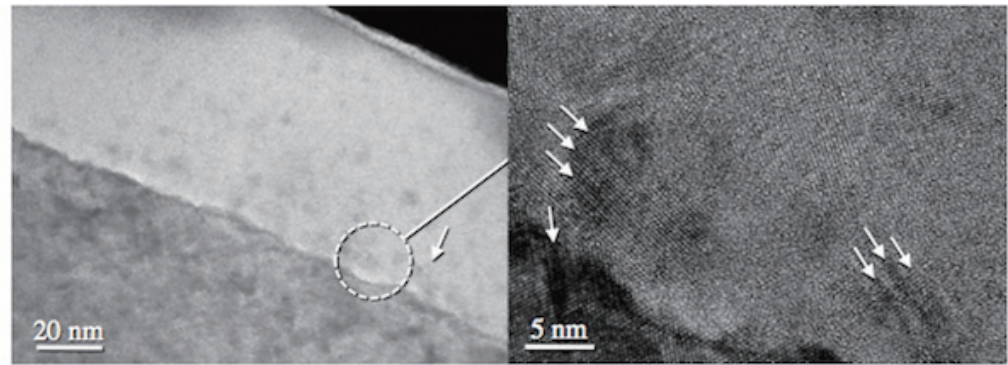

(c)

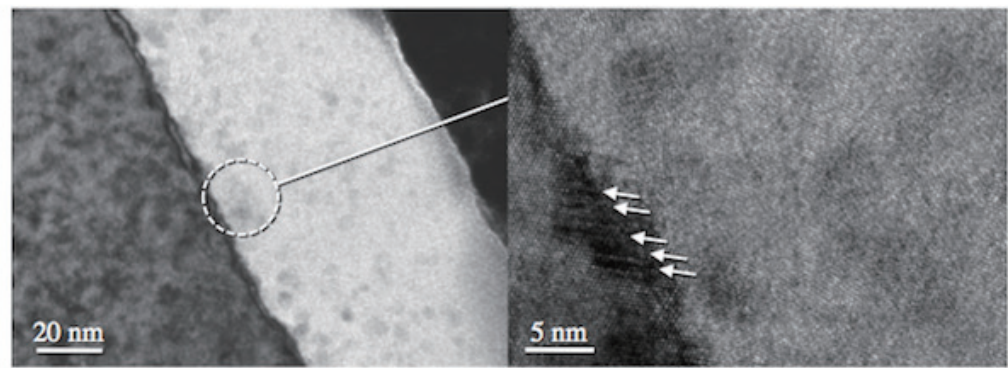

(d)

Fig. 9. TEM images of Si substrate before and after $700{ }^{\circ} \mathrm{C}$ annealing: (a) FIB-irradiated substrate, and substrates annealed for (b) $10 \mathrm{~s}$, (c) $5 \mathrm{~min}$, and (d) $60 \mathrm{~min}$. The white arrows in TEM images indicate Moiré fringes that are localized crystal rotations. Images on the right are enlargements of the circled area in corresponding images on the left.

close to the ideal value upon annealing. Also, the roughened surface with the formation of Ga droplets by annealing and the large strain induced by misorientations in recrystallized portions might have reduced the strength. In the future, if we optimize the annealing conditions to 


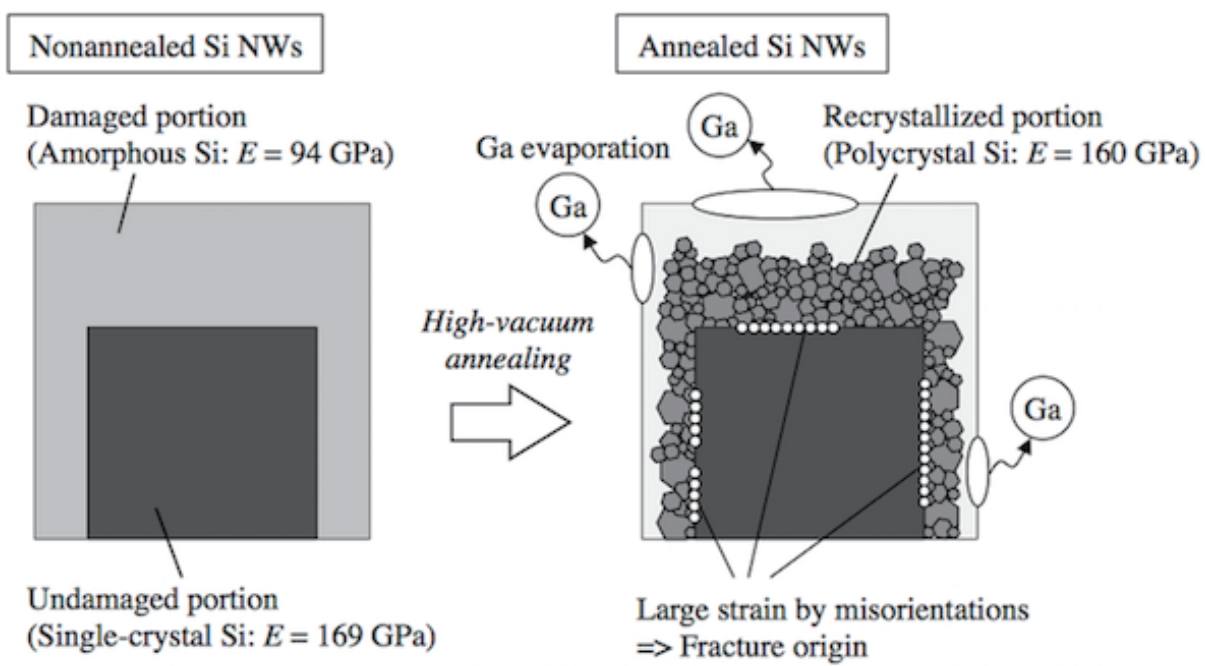

Fig. 10. Crystallinity-damage recovery and Ga annihilation processes affecting recovery of the mechanical characteristics.

completely recrystallize the FIB-induced amorphous structure to a single-crystal structure, very strong Si nanostructures could be fabricated using the combination of FIB direct fabrication and vacuum annealing techniques.

\section{Conclusions}

In this research, we investigated the effects of high-vacuum annealing on the mechanical properties of Ga-implanted Si NWs. The combination of a MEMS-based uniaxial tensile testing device and FIB-based sampling techniques enabled us to accurately measure Young's modulus and fracture strength by in situ SEM. All the Si NWs showed an almost linear stressstrain relationship until fracture, indicating that the NWs fractured in a brittle manner during elastic deformation. Young's modulus gradually approached the ideal value of SCS (001)[110] with increasing annealing time because of the recovery of the crystallinity of the FIBdamaged portion, whereas the fracture strength dropped first at $10 \mathrm{~s}$ of annealing and then slightly increased with annealing time because of the roughened surface and the formation of Ga droplets, which are stress concentration sites. By SEM and TEM observations and EDX analysis, the implanted $\mathrm{Ga}^{+}$ions were found to be completely removed by high-vacuum annealing at $700{ }^{\circ} \mathrm{C}$ for $10 \mathrm{~min}$, the FIB-induced surface damage gradually recrystallized from the interface near the SCS substrate, and the implanted Ga atoms evaporated while forming island-shaped Ga-rich platelets on the Si NWs. From the above findings, clarifying the repair mechanism of the FIB-induced damage upon high-vacuum annealing will be useful for the realization of Si nanostructures with perfect crystals and surfaces for MEMS/NEMS. 


\section{References}

1 J. S. Hyun, J.-S. Moon, J.-H. Park, J. W. Kim, Y. D. Kim, and J.-H. Boo: Mater. Sci. Eng., B 149 (2008) 292.

2 A. Uranga, J. Verd, E. Marigo, J. Giner, J. L. Munoz-Gamarra, and N. Barniol: Sens. Actuators, A 197 (2013) 88.

3 L. Boodhoo, L. Crudgington, H. M. H. Chong, Y. Tsuchiya, Z. Moktadir, T. Hasegawa, and H. Mizuta: Microelectron Eng. 145 (2015) 66.

4 X. L. Feng, R. He, P. Yang, and M. L. Roukes: Nano Lett. 7 (2007) 1953.

5 C. Mu, Q. Zhao, D. Xu, Q. Zhuang, and Y. Shao: J. Phys. Chem. B 111 (2007) 1491.

6 Y. Wu, Y. Cui, L. Huynh, C. J. Barrelet, D. C. Bell, and C. M. Lieber: Nano Lett. 4 (2004) 433.

7 A. C. Fischer, L. M. Belova, Y. G. M. Rikers, B. G. Malm, H. H. Radamson, M. Kolahdouz, K. B. Gylfason, G. Stemme, and F. Niklaus: Adv. Funct. Mater. 22 (2012) 4004.

8 S. Matsui, T. Kaito, J. Fujita, M. Komuro, K. Kanda, and Y. Haruyama: J. Vac. Sci. Technol., B 18 (2000) 3181.

9 V. Giliberti, E. Sakat, L. Baldassarre, A. D. Gaspare, A. Notargiacomo, E. Giovine, J. Frigerio, G. Isella, M. Melli, A. W. Bargioni, S. Aloni, S. Sassolini, S. Cabrini, P. Biagioni, M. Ortolani, and M. Bollani: Microelectron. Eng. 141 (2015) 168.

10 Z. Wang, T. Kato, T. Hirayama, N. Kato, K. Sasaki, and H. Saka: Appl. Surf. Sci. 241 (2005) 80.

11 T. Fujii, T. Namazu, K. Sudoh, S. Sakakihara, and S. Inoue: J. Eng. Mater. Technol. 135 (2013) 041002.

12 N. Ikarashi and K. Watanabe: Jpn. J. Appl. Phys. 39 (2000) 1278.

13 A. Barna, L. Toth, B. Pecz, and G. Radnoczi: Microsc. Semicond. Mater. 157 (1997) 479.

14 Y. Yabuuchi, S. Tametou, T. Okano, S. Inazato, S. Sadayama, Y. Yamamoto, K. Iwasaki, and Y. Sugiyama: J. Electron Microsc. 53 (2004) 471.

15 C. H. Chu, Y. F. Hsieh, L. R. Harriott, and H. H. Wade: J. Vac. Sci. Technol., B 9 (1991) 3451.

16 T. Nagata, Y. Sakuma, M. Haemori, K. Nakajima, R. Kometani, K. Kanda, S. Matsui, and T. Chikyow: Jpn. J. Appl. Phys. 47 (2008) 9010.

17 A. Schilling, T. Adams, R. M. Bowman, and J. M. Gregg: Nanotechnol. 18 (2007) 035301.

18 M. Ishida, J. Fujita, T. Ichihashi, and Y. Ochiai: J. Vac. Sci. Technol., B 21 (2003) 2728.

19 T. Fujii, K. Sudoh, S. Sakakihara, M. Naito, S. Inoue, and T. Namazu: Jpn. J. Appl. Phys. 52 (2013) 110118.

20 T. Fujii, K. Sudoh, S. Inoue, and T. Namazu: Sens. Mater. 28 (2016) 89.

21 K. Sudoh, H. Iwasaki, R. Hiruta, H. Kuribayashi, and R. Shimizu: J. Appl. Phys. 105 (2009) 083536.

22 I. Mizushima, T. Sato, S. Taniguchi, and Y. Tsunashima: Appl. Phys. Lett. 77 (2000) 3290.

23 J. J. McSkimin: J. Appl. Phys. 24 (1953) 988.

24 T. Namazu, Y. Isono, and T. Tanaka: J. Microelectromech. Syst. 9 (2000) 450.

25 K. Sato, T. Yoshika, T. Ando, M. Shikida, and T. Kawabata: Sens. Actuators, A 70 (1998) 148.

26 J. Mayer, L. A. Giannuzzi, T. Kamino, and J. Michael: MRS Bull. 32 (2007) 400.

27 T. Tsuchiya, O. Tabata, J. Sakata, and Y. Taga: J. Microelectromech. Syst. 7 (1998) 106.

28 J. P. Cui, Y. L. Hao, S. J. Li, M. L. Sui, D. X. Li, and R. Yang: Phys. Rev. Lett. 102 (2009) 045503.

29 M. Wu, A. Trampert, T. A. Zoubi, M. Benyoucef, and J. P. Reithmaier: Acta Mater. 90 (2015) 133. 